\title{
Investigation of an outbreak of group A Streptococcus in a Regina retirement residence and personal care home, 2018
}

\author{
Trecker M A, Danielson C, Koutsoulis G, Lloyd K, Benz Tramer C, Diener T, Hennink M \\ Population and Public Health Services, Saskatchewan Health Authority, Regina, SK.
}

Corresponding author:

Molly Trecker (molly.trecker@saskhealthauthority.ca)

\begin{abstract}
Streptococcus pyogenes (group A Streptococcus) is a common bacterium that causes infections ranging from minor illnesses, like strep throat, to life-threatening invasive disease. The elderly are particularly at risk of invasive infection, with this risk compounded by living in communal settings, including long-term care facilities or personalcare homes. Following the identification of five invasive group A streptococcal infections in residents of a Regina retirement residence and personal care home over a period of five months, an outbreak was declared on May 8, 2018. Over the 10 weeks the outbreak lasted, 10 cases were diagnosed, attributable to nine individuals: six residents and three staff. Five of the 10 cases $(50 \%)$ were invasive, all of which required hospitalization. The predominant emm type was $92-$ a type not common in Canada. Interventions, including onsite inspections, weekly surveillance, hand hygiene and environmental cleaning improvements, as well as mass screening for carriage of group A Streptococcus were carried out in collaboration with the personal-care home. Mitigating outbreak risks in private retirement residences and personal care homes requires that facilities establish robust infection control programs, including hand hygiene and effective environmental cleaning, and work collaboratively with Public Health officials to address outbreaks.
\end{abstract}

KEYWORDS

Group A Streptococcus; Streptococcus pyogenes; iGAS; GAS; personal care home

Group A streptococcal (GAS) infections are caused by a common bacterium, Streptococcus pyogenes. Infections are often mild, manifesting as illnesses like strep throat, and typically respond well to treatment. Invasive disease (iGAS) can occur, however, causing life-threatening conditions such as necrotizing fasciitis or streptococcal toxic shock syndrome. The elderly are particularly vulnerable to iGAS infection [1], and have the highest case-fatality rates [2]. A number of medical conditions have also been found to be associated with increased risk of iGAS, including dermatologic conditions (such as bullous pemphigoid), diabetes, heart disease, and cancer - conditions more common among this demographic. Further, the risk of acquiring GAS is compounded by living in crowded settings, such as long-term care facilities (LTCFs) or personal care homes ( $\mathrm{PCHs}$ ) [3], and there is a substantial amount of evidence related to the risks specific to residents of these facilities [4-6].

Although the related burden of disease and number of deaths is lower in developed countries such as Canada, iGAS is a nationally notifiable disease [7]. In Saskatchewan, an average of 87 iGAS cases was reported annually between 2004 and 2017. Cases occurred at the same rate among males and females, and $21 \%$ of cases were 65 years of age and over. The most prominent emm types ${ }^{1}$ were emm81, emm1, and emm41.11. The majority of cases presented as bacteremia, with a very small proportion being necrotizing fasciitis (Saskatchewan Ministry of Health, personal communication).

Located in south-central Saskatchewan, Regina is the capital city, with a population of 214,631 individuals, $13.8 \%$ of whom are 65 years of age or older [8]. We report here on an outbreak of GAS, which occurred in a dual private retirement residence and $\mathrm{PCH}$ in Regina in the late winter till the spring of 2018. The classifications of LTCFs and PCHs differ from one another in that in Saskatchewan, LTCFs are part of the publicly funded healthcare system, and tend to serve residents with more substantial care needs, while $\mathrm{PCH}$ are privately operated facilities, licensed by the Ministry of Health.

This outbreak occurred in a facility that serves as both a private retirement residence and $\mathrm{PCH}$, housing 199 residents, with 50 staff. The multi-story building includes both independent living suites and a $\mathrm{PCH}$. Services provided by the $\mathrm{PCH}$ include

\section{ACKNOWLEDGEMENTS}

We would like to acknowledge the collaboration of the facility during this outbreak.

\section{CONFLICTS OF INTEREST}

None.

${ }^{1}$ emm sequence typing is a system used to characterize the degree of genetic diversity among circulating strains of $S$. pyogenes; emm types are numerical, and, where applicable, the subtype is indicated by a number following the decimal point. 
assistance with all activities of daily living, and basic nursing care is provided by Licensed Practical Nurses. Residents, including those that live in the $\mathrm{PCH}$, are able to move about the building via two elevators - one in the south wing and one in the north wing. In February 2018, a new male resident moved into the $\mathrm{PCH}$ upon discharge from hospital, where he had been treated for iGAS disease (bacteremia) since December 2017. He would ultimately prove to be the index case of the outbreak.

For the purposes of describing this outbreak, the following case definitions were used. Cases were defined as those with laboratory-confirmed GAS from any site, with or without symptoms. iGAS was defined as isolation of GAS (S. pyogenes) from a normally sterile site, such as blood [9]. Persistently positive cases were those who remained positive for GAS despite appropriate antibiotic therapy. A case was considered to be a repeat infection when an individual was treated for GAS, confirmed to be negative for GAS from all sites post-treatment, and subsequently developed another symptomatic infection with GAS isolation.

On February 2, 2018, the index case of the outbreak was discharged from hospital, where he had been treated for iGAS (blood). He moved into the North wing of the PCH located on the 2nd floor. On March 9, a second case of iGAS (blood) was identified in a resident on a different floor. A third case (blood) followed two days later, on March 11, in a resident living in the same $\mathrm{PCH}$ as the index case. At this time, a four-week period of surveillance for GAS was initiated. No new cases were identified among residents and staff during the four-week period, and surveillance was ended on April 10. Between May 3 and 8, however, two new iGAS (blood) cases were identified among residents of the building, including one living in the $\mathrm{PCH}$. At the same time, the index case was re-hospitalized for a cutaneous GAS infection. In light of these new cases, an outbreak was declared on May 8.

All invasive GAS isolates are submitted to the National Microbiology Laboratory for typing and results are available within approximately one month. emm typing from the index case identified in December 2017, and the two cases identified in March 2018, revealed the same type (emm92), suggesting they could potentially represent the same strain. Unfortunately, however, this cannot be confirmed because strain typing information was unavailable. Screening of staff and residents was carried out to identify any new cases. In total, all 50 staff, and 25 residents living in the north wing $\mathrm{PCH}$, were swabbed (nose, throat, and any open wounds). Three staff members were found to have asymptomatic carriage of GAS in their throats, and one resident had a GAS-positive wound. The three staff were started on Cephalexin 500mg QID for 10 days by their family physicians, per the Guidelines for the Prevention and Control of Invasive Group A Streptococcal Disease [9], and excluded from work until 48 hours after starting the antibiotics. The resident with the positive wound swab was also treated with antibiotics, and was maintained on contact and droplet precautions for 48 hours after treatment initiation. Each case found during mass screening and/or surveillance was rescreened at 14 and 28 days after treatment began.
The initial on-site environmental inspection was carried out by the communicable disease control team made up of Public Health nurses and a Public Health inspector. It included a tour of the facility, assessment of hand hygiene practices, personal protective equipment (PPE) use, and environmental cleaning processes. The inspection revealed a lack of access to hand-washing sinks and alcohol-based hand rub (ABHR), insufficient use of PPE and related isolation procedures, and that environmental cleaning products were not being applied per manufacturers' recommendations. Links to resources from Public Health Ontario, Public Health Agency of Canada (PHAC), and the Patient Safety Institute were provided to assist the management of the $\mathrm{PCH}$ to establish infection control procedures, including PPE use and appropriate isolation measures for their facility.

The primary focus of the response was to improve access to $\mathrm{ABHR}$, reinforce the need for hand hygiene among staff, and address deficiencies in environmental cleaning practices. In total, eight on-site visits were made over the course of the outbreak to support and encourage the adoption of the advised practices, and to assess progress in this area. Public Health staff provided a 'train the trainer' session to facilitate a review with all staff of how and when they should be cleaning their hands, utilizing resources from the local health region and the Hand Hygiene Practices in Healthcare Settings document from PHAC [10]. Addressing the lack of access to hand-washing sinks and $A B H R$ was a priority to help curb the spread. Installation of wall-mounted ABHR dispensers and provision of staff with small bottles of ABHR to carry with them was recommended. The $\mathrm{PCH}$ management voiced concerns, however, that mounting ABHR dispensers would diminish the 'home-like' feeling that they had strived to create, and the unexpected cost delayed the installation of these until the end of the outbreak. Staff were provided with personalsized bottles of $A B H R$ and facility management initiated a process for auditing whether staff were carrying and using these. When the public health team interviewed staff about their hand hygiene practices, staff reported that they were 'scared' because of the outbreak and cleaning their hands more often; however, none of the staff interviewed were carrying the personal size bottles of $\mathrm{ABHR}$ at the time.

Steps to address deficiencies in environmental cleaning involved working with the facility cleaning staff and management to ensure the cleaning products were being applied at the right concentration for disinfecting, and that the recommended wet contact time was observed. Increased attention was paid to disinfection of high-touch surfaces in the common areas and in the GAS-positive resident rooms. Public Health worked with management of the $\mathrm{PCH}$ to create checklists of the high-touch surfaces, which were to be cleaned on day and night shifts. The use of chemical test strips and a recording log was advised to test and track that the cleaning products were being dispensed at the correct concentration, but this was not done consistently. Testing by Public Health staff during site visits, however, found that the correct concentration of chemical was present on the housekeeping carts in use on the unit. Environmental sampling was not done.

From December 2017, when the index case was in hospital, through June 2018, 10 cases of GAS were diagnosed with links 
to the facility (attack rate $=3.0 \%$ among residents). In addition to the index case, there were two cases in March, six in May, and one in June (Figure 1). The 10 cases were attributable to nine individuals - six residents and three staff. Of the six residents and three staff involved in the outbreak, five (55.6\%) were female and four (44.4\%) were male. The mean age was 91.5 years among residents, and all were over 80 years of age.

The index case was found to be persistently positive with GAS despite appropriate antibiotic treatments, and another resident was positive for GAS on two separate occasions (repeat infection).

Five of the 10 cases (50.0\%), all among residents, were invasive, requiring hospitalization. Three of the five non-invasive cases $(60.0 \%)$ were among asymptomatic staff, identified upon screening. Eight of the 10 cases $(80.0 \%)$ were type emm92; the remaining two cases $(20.0 \%)$ - both among asymptomatic staff were emm 1.0 and emm1.41. (Table 1)

Site visits and interviews revealed that the six resident cases (all emm92) lived in the north wing of the building. Cases occurred on all floors of the building, with a significant clustering of four residents in the $\mathrm{PCH}$. The four rooms in the
$\mathrm{PCH}$ were located in close proximity to one another, and to the north elevator (Figure 2). Indirect contact between the index case and others occurred on various occasions while riding in the north elevator at the same time. The one staff case with emm92 had an office in the north wing. This staff member reported indirect contact with the index case and direct contact with two of the iGAS cases. Direct contact occurred after onset of symptoms in the two cases when she was called to assist when each became ill, requiring transfer to hospital, where each was diagnosed with iGAS bacteremia.

All but two cases were negative on repeat screens at 14 and 28 days after treatment initiation. The index case was persistently positive on four different occasions, and one other resident had positive wounds on two occasions (repeat infection).

This was the first GAS outbreak to occur in a private $\mathrm{PCH}$ in the Regina area. Because PCHs are privately owned and operated, there are varying degrees of formal infection control programs, and a collaborative approach to identification of and response to the outbreak was required. We found the primary challenges to outbreak control were lack of compliance with

FIGURE 1: Timeline of GAS outbreak at a Regina Personal Care Home, December 2017 to June 2018. ( $R=$ resident, $S=$ staff)

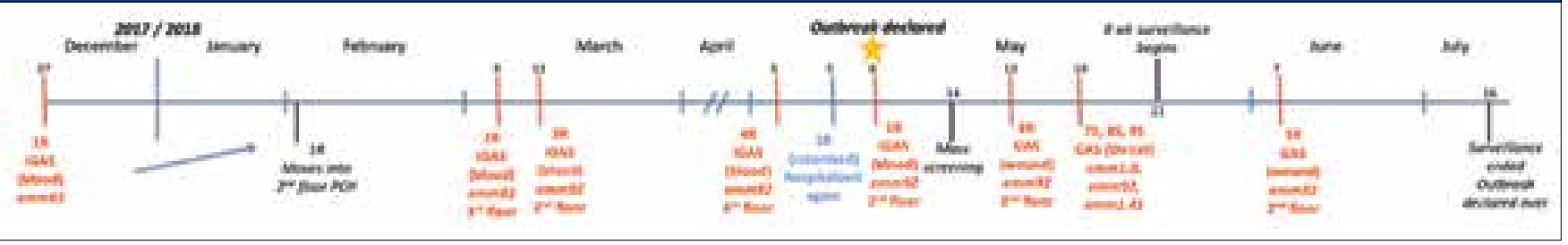

\section{TABLE 1: Characteristics of 10 cases of GAS among nine individuals at the Regina PCH (includes one repeat infection).}

( $R=$ resident, $S=$ staff)

\begin{tabular}{|c|c|c|c|c|c|}
\hline Case number & ID & New or repeat & Invasive (bacteremia) & Hospitalized & emm type \\
\hline 2 & $2 \mathrm{R}$ & New & $\mathrm{Y}$ & Y & 92 \\
\hline 3 & $3 R$ & New & Y & Y & 92 \\
\hline 4 & $4 \mathrm{R}$ & New & $\mathrm{Y}$ & $\mathrm{Y}$ & 92 \\
\hline 7 & $7 \mathrm{~S}$ & New & $\mathrm{N}$ & $\mathrm{N}$ & 1.0 \\
\hline 8 & $8 S$ & New & $\mathrm{N}$ & $\mathrm{N}$ & 92 \\
\hline 9 & $9 \mathrm{~S}$ & New & $\mathrm{N}$ & $\mathrm{N}$ & 1.41 \\
\hline 10 & $5 \mathrm{R}$ & Repeat & $\mathrm{N}$ & $\mathrm{N}$ & 92 \\
\hline
\end{tabular}

FIGURE 2: Diagram illustrating the proximity of cases' rooms (yellow) in the North Wing.

Patient room

Patient room iGAS

North Hallway of the $\mathrm{PCH}$, 2nd Floor
Patient room

+ GAS $\times 2$
Patient room

\begin{tabular}{|c|c|c|c|c|}
\hline \multicolumn{5}{|c|}{ North Hallway of the PCH, 2nd Floor } \\
\hline Patient room & Storage & $\begin{array}{c}\text { Patient room } \\
+ \text { GAS wound }\end{array}$ & $\begin{array}{c}\text { Patient room } \\
\text { iGAS } \\
\text { Index Case }\end{array}$ & North Elevator \\
\hline
\end{tabular}


proper hand hygiene, and lack of proper implementation of PPE, isolation procedures, and environmental cleaning. The lack of adherence to routine practices, like hand hygiene and/or environmental cleaning, likely contributed to GAS transmission. The spatial clustering of seven individuals (comprising eight cases of infection) with emm92 either living or working in the north wing of the building also supports the possibility of personto-person transmission, given the common environment and opportunities for contact. Such spatial clustering of cases within a facility has been previously reported [11]. Unfortunately, since strain typing information was not available, we cannot definitively say that these cases shared the same strain. Propagation of infection by staff within such facilities, along with poor infection control measures, has also been documented as potentially contributing to outbreaks [5]. In this scenario, the outbreak was eventually brought under control when access to hand hygiene materials, appropriate environmental cleaning processes, and use of proper PPE were fully established, further supporting the hand hygiene/environmental contamination hypothesis.

An additional element that likely contributed to this outbreak is the fact that the index case had an underlying dermatological condition that predisposed him to remain colonised with GAS in spite of treatment. Because patients such as this are unlikely to be able to be decolonised, strict infection control process are needed. Such strict controls were not in place in the $\mathrm{PCH}$ at the time the index case became a resident, which likely led to environmental contamination and person-to-person spread, most likely by staff at the facility.

Another interesting element of this outbreak was the prevalence of the emm92 type. emm92 is uncommon both nationally and in the province. In Canada, emm 1 has generally been the most prevalent emm type [12]. Types emm81 (17\%), emm1 (11\%), and emm41.11 (8\%) are the most common in Saskatchewan (Saskatchewan Ministry of Health, personal communication). In the five-year period from 2013-2018, emm92 was identified in the Regina area only four times previous to this outbreak - twice in 2016 and twice in 2017. No epi-link was identified for the index case, and the source of his initial infection with emm92 remains unknown.

Facilities where elderly residents live together, such as retirement residences or $\mathrm{PCHs}$, with many vulnerable persons living in close proximity, provide an ideal environment for disease transmission. Mitigating this risk requires that facilities establish robust infection control programs, including hand hygiene and effective environmental cleaning. The implication of poor hand hygiene and limited infection control procedures as factors contributing to this outbreak highlight important areas of focus for such facilities. Unlike publicly funded LTCFs, PCHs are privately owned, and operate with no mandatory standards for infection control. Because of this, a collaborative approach between Public Health and PCHs is necessary to ensure the wellbeing of residents of such private facilities when outbreaks occur.

\section{REFERENCES}

1. Steer AC, Lamagni T, Curtis N, Carapetis JR. (2012). Invasive group A streptococcal disease. Drugs, 72(9), 12131227. doi:10.2165/11634180-000000000-00000

2. Centers for Disease Control and Prevention. (2018). ABCs report: Group A Streptococcus, 2016. Retrieved from: https://www.cdc. gov/abcs/reports-findings/survreports/gas16.html.

3. Factor SH, Levine OS, Schwartz B, Harrison LH, Farley MM, McGeer A, et al. (2003). Invasive group A streptococcal disease: risk factors for adults. Emerging Infectious Diseases, 9(8), 970977. doi:10.3201/eid0908.020745

4. High KP, Jordan HT, Richards Jr CL, Burton DC, Thigpen MC, Van Beneden CA. (2007). Group A streptococcal disease in long-term care facilities: descriptive epidemiology and potential control measures. Clinical Infectious Diseases, 45(6), 742-752. doi:10.1086/520992

5. Nanduri SA, Metcalf BJ, Arwady MA, Edens C, Lavin MA, Morgan J, et al. (2019). Prolonged and large outbreak of invasive group A Streptococcus disease within a nursing home: repeated intrafacility transmission of a single strain. Clinical Microbiology and Infection, 25(2), 248-e1. doi:10.1016/j.cmi.2018.04.034

6. Saavedra-Campos M, Simone B, Balasegaram S, Wright A, Usdin M, Lamagni T. (2017). Estimating the risk of invasive group A Streptococcus infection in care home residents in England, 2009-2010. Epidemiology \& Infection, 145(13), 2759-2765. doi:10.1017/S0950268817001674

7. Efstratiou A, Lamagni T. (2017). Epidemiology of Streptococcus pyogenes. In Streptococcus pyogenes: basic biology to clinical manifestations [Internet]. University of Oklahoma Health Sciences Center.

8. Statistics Canada. (2019). Census Profile, 2016 Census. Retrieved November 6, 2019, from https://www12.statcan.gc.ca/censusrecensement/2016/dp-pd/prof/index.cfm?Lang=E.

9. Public Health Agency of Canada. (2009). Invasive group A streptococcal., Retrieved November 6, 2019 from https://www. canada.ca/en/public-health/services/reports-publications/canadacommunicable-disease-report-ccdr/monthly-issue/2009-35/ definitions-communicable-diseases-national-surveillance/ invasive-group-streptococcal.html.

10. Public Health Agency of Canada. (2013). Hand hygiene practices in healthcare settings. Retrieved November 6, 2019 from http:// publications.gc.ca/site/eng/430135/publication.html.

11. Arnold KE, Schweitzer JL, Wallace B, Salter M, Neeman R, Hlady WG, et al. (2006). Tightly clustered outbreak of group a streptococcal disease at a long-term care facility. Infection Control \& Hospital Epidemiology, 27(12), 1377-1384. doi:10.1086/508820

12. Public Health Agency of Canada. (2017). National laboratory surveillance of invasive streptococcal disease in Canada - annual summary 2015. Retrieved November 6, 2019 from https://www. canada.ca/en/public-health/services/publications/drugs-healthproducts/national-laboratory-surveillance-invasive-streptococcaldisease-canada-annual-summary-2015.html. 